\title{
Stretching Force Constant of the Carbonyl Bond in Unconjugated Ketones
}

\author{
J. O. HALFORD \\ Department of Chemistry, University of Michigan, Ann Arbor, Michigan
}

(Received July 18, 1955)

\begin{abstract}
The secular equation derived for the normal frequencies of a $\mathrm{Y}$-shaped $\mathrm{C}_{2} \mathrm{CO}$ model can be used with fair accuracy to obtain the carbonyl bond force constant from the measured carbonyl frequency of any symmetrical ketone if the carbon-carbon force constant and the bond angles are known or can be reasonably assigned.

The narrow spread of carbonyl frequencies observed for unconjugated ketones, with assumed "normal" angles and carbon-carbon force constant, indicates a nearly constant carbonyl stretching force constant in the range $10.2 \pm 0.3\left(\times 10^{5}\right.$ dynes $\left./ \mathrm{cm}\right)$. This value should be equally reliable for unsymmetrical unconjugated ketones.

In formaldehyde, the calculated force constant is usually above 12.5 units.
\end{abstract}

$S^{\mathrm{T}}$ TRETCHING force constants for the carbonyl $\mathcal{S}$ bond have been calculated from the experimental vibration frequencies of several of the simpler carbonyl compounds. For example, Branch and Calvin ${ }^{1}$ have tabulated four values, 13.4, 14.2, 15.3, and $19.0\left(\times 10^{5}\right.$, dynes $/ \mathrm{cm}$ ) for, respectively, formaldehyde, carbonyl sulfide, carbon dioxide, and carbon monoxide. Of these compounds, carbon monoxide obviously does not contain a representative carbonyl bond, and, in carbonyl sulfide and carbon dioxide, resonance effects would be expected to account for deviations from representative properties. If there is a nearly constant stretching force constant for the carbonyl bond in the absence of resonance, only formaldehyde from the above list could yield a good approximation to the true value. Since, however, the first member of a homologous series often has properties different from the heavier members, even the force constant obtained from the formaldehyde frequencies may not be representative.

According to a tabulation by F. A. Miller, ${ }^{2}$ the carbonyl frequencies of unconjugated aliphatic ketones, presumably noncyclic, lie within the narrow range from 1705 to $1720 \mathrm{~cm}^{-1}$. Since the frequency is expressible as a function of the force constants, atomic masses, and the characteristic bond angles, this narrow range suggests fixed force constants and angles together with insensitivity to the masses. However, the possibility that there is a fortuitous cancellation of variations through the series must not be dismissed at the outset. The value 1686 obtained by Stiles ${ }^{3}$ for $t$. butyl ketone shows that such variations can occur. For aldehydes, except formaldehyde, the observed frequencies are usually between 1650 and $1730 \mathrm{~cm}^{-1}$.

Interpretation of the data requires the development of a practical and accurate enough expression for the carbonyl frequency as a function of the masses, angles, and force constants. If a potential energy function is assumed, such a relation can always be written in the

\footnotetext{
${ }^{1}$ G. E. K. Branch and M. Calvin, The Theory of Organic Chemistry (Prentice-Hall, Inc., New York, 1941), p. 154.

2 H. Gilman, Organic Chemistry (John Wiley and Sons, Inc., New York, 1953), Vol. III, p. 140.

8. M. Stiles, Dissertation, Harvard University, 1953.
}

form of a vanishing secular determinant derived by the well-established procedures of the normal coordinate treatment.

For all cases except formaldehyde, the solution of the complete secular determinant is inconveniently detailed. In the most general case the problem is hopelessly complicated. For rigid symmetrical ketones, for which the parallel frequencies factor out as a separate problem, the complexity is at a minimum, but too much algebraic and arithmetical detail is still involved.

It is therefore desirable in all cases to seek some simplified model which can be substituted for the more detailed complete molecular model to give the relation between frequency and force constant without serious loss of accuracy. That there is such a model can be inferred from the nearly constant carbonyl frequency of unconjugated ketones. For a symmetrical ketone, there should always be a $\mathrm{Y}$-shaped model $\mathrm{X}_{2} \mathrm{CO}$ with the same XCX angle and the same carbonyl force constant as the molecular model, which will give the same carbonyl frequency. If the force constant for stretching of the $\mathrm{C}-\mathrm{X}$ bond is set at a reasonable single bond value, the required carbonyl frequency can be obtained with a proper choice of the mass of $\mathrm{X}$. The simplified $\mathrm{X}_{2} \mathrm{CO}$ model can therefore be substituted for the complete molecular model if a basis can be found for assigning the mass of $\mathrm{X}$.

It develops that the maximum or carbonyl frequency of a model $\mathrm{X}_{2} \mathrm{CO}$ does not change much as the mass of $\mathrm{X}$ is increased from 10 atomic weight units to infinity, and that the corresponding frequency of a symmetrical planar model $(M C)_{2} \mathrm{CO}$, in which the $M \mathrm{C}$ linkage is a normal single bond, is insensitive to variation of the mass or position of $M$. This suggests the substitution of the simple model $\mathrm{C}_{2} \mathrm{CO}$, with the appropriate $\mathrm{CCC}$ angle, for each of the symmetrical ketones.

This proves to be a more satisfactory approximation than the alternative one in which the $R$ groups of $R_{2} \mathrm{CO}$ would be regarded as rigid and would be treated as single masses attached to the carbonyl group by normal carbon-carbon single bonds. In such a model an increase in the mass of $R$ would produce a small decrease 
in the carbonyl frequency. In the examples described below, it will be found in every case that the extension of a model by the attachment of a particle by a normal single bond increases the maximum frequency. To the degree that this effect is general, it will always be more accurate to ignore the extension than to combine the added particle rigidly with the mass to which it is attached.

The occurrence of an increase of the maximum frequency with extension of the model is nicely illustrated by the "linear crystal" consisting of a row of $N$ equal point masses connected by uniform bonds of force constant $k .{ }^{4}$ For this linear model the maximum frequency is:

$$
\nu=\left[k /\left(\pi^{2} m\right)\right]^{t} \sin [(N-1) \pi /(2 N)] .
$$

The maximum frequency increases by successively smaller increments as new masses are added to the chain. If, however, all masses beyond the second were assumed to constitute a rigid radical without internal motion, each mass added to this radical would cause a decrease in the calculated frequency. It is evident that less error is introduced by ignoring the added masses than by the rigid radical assumption. This proves to be true also for the $\mathrm{Y}$-shaped model $\mathrm{C}_{2} \mathrm{CO}$.

The first step in justifying the proposed substitution has been carried out by Burkard, ${ }^{5}$ who studied the frequencies of a model $\mathrm{X}_{2} \mathrm{CO}$ with a fixed $\mathrm{XCX}$ angle and force constants as a function of the mass of $X$. Burkard found that the change of frequency with mass decreases rapidly as the mass is increased. The maximum parallel frequency, for $\mathrm{X}=\mathrm{H}$, is in the 3000 $\mathrm{cm}^{-1}$ region characteristic of the $\mathrm{CH}$ bond. As $\mathrm{X}$ increases in mass, the frequency rapidly decreases up to masses of 10 or 12 units and thereafter changes very little as the mass is increased to infinity. At the formaldehyde level, with $\mathrm{X}$ at one unit, the carbonyl frequency is the second, rather than the highest parallel frequency, and the motion represented has different phase relations between the atomic displacements from the models with masses above 10 units.

For each of the normal parallel vibrations, Burkard has studied the distribution of energy among the several terms of his potential energy equation. He finds that the angular or bending forces have little effect upon the highest frequency of the model. Consequently the rela* tion between the carbonyl force constant and frequency is substantially independent of the magnitude of the bending forces and the assumed form of their contribution to the potential energy.

A simple three constant potential energy function, chosen to minimize the detail of calculation, has therefore been used in the present study for the potential energy in the parallel motions of the symmetrical model

\footnotetext{
4J. O. Halford, J. Chem. Phys. 19, 1375 (1951).

50 . Burkard, Proc. Indian Acad. Sci. 8, 365 (1938).
}

TABLE I. Highest parallel frequency of the symmetrical model $\mathrm{X}_{2} \mathrm{CO}$ as function of the mass of $\mathrm{X}$ at several $\mathrm{XCX}$ angles.

\begin{tabular}{|c|c|c|c|c|c|}
\hline \multicolumn{6}{|c|}{$\triangle \mathrm{XCX}$ angle } \\
\hline$m_{x} \backslash$ & 0 & $60^{\circ}$ & $90^{\circ}$ & $120^{\circ}$ & $180^{\circ}$ \\
\hline 1 & 3030 & 2970 & 2906 & 2838 & 2764 \\
\hline 12 & 1888 & 1825 & 1761 & 1695 & 1629 \\
\hline 96 & 1834 & 1781 & 1728 & 1676 & 1626 \\
\hline$\infty$ & 1829 & 1777 & 1725 & 1675 & 1626 \\
\hline
\end{tabular}

$\mathrm{X}_{2} \mathrm{CO}$. The potential function follows:

$$
\begin{aligned}
2 V=k_{1}\left(x_{2}-x_{1}\right)^{2}+2 k_{2}\left(\left[x_{3}-x_{2}\right] \cos \theta+y_{3} \sin \theta\right)^{2} \\
+2 k_{3}\left(\left[x_{2}-x_{3}\right] \sin \theta+y_{3} \cos \theta\right)^{2} .
\end{aligned}
$$

In Eq. (2), $x_{1}$ is the displacement of the oxygen atom, $x_{2}$ is the displacement of the carbon, and $x_{3}$ and $y_{3}$ are displacements of the $\mathrm{X}$ units. The carbonyl and $\mathrm{C}-\mathrm{X}$ stretching force constants are represented, respectively, by $k_{1}$ and $k_{2}$, while $k_{3}$ is the restoring force for unit relative displacement of $\mathrm{C}$ and $\mathrm{X}$ perpendicular to the $\mathrm{CX}$ bond. $\theta$ is half the XCX angle.

The secular equation in $\lambda\left(=4 \pi^{2} c^{2} \nu^{2}\right.$, for $\nu$ in wave numbers) for the three parallel frequencies has the following form:

$$
\begin{aligned}
& \lambda^{3}-\left(k_{1}\left[m_{1}+m_{2}\right] / m_{1} m_{2}+\left[k_{1}+k_{3}\right] / m_{x}\right. \\
& \left.\quad+2\left[k_{2} \cos ^{2} \theta+k_{3} \sin ^{2} \theta\right] / m_{2}\right) \lambda^{2} \\
& \quad+\left(k_{1}\left[k_{2}+k_{3}\right] / m_{1} m_{x}+k_{1}\left[k_{2}+k_{3}\right] / m_{2} m_{x}+k_{2} k_{3} / m_{x}^{2}\right. \\
& \left.\quad+2 k_{2} k_{3} / m_{2} m_{x}+2 k_{1}\left[k_{2} \cos ^{2} \theta+k_{3} \sin ^{2} \theta\right] / m_{1} m_{2}\right) \lambda \\
& \quad-k_{1} k_{2} k_{3}\left[m_{1}+m_{2}+2 m_{x}\right] / m_{1} m_{2} m_{x}^{2}=0 .
\end{aligned}
$$

In Eq. (3), $m_{1}, m_{2}$, and $m_{x}$ are, respectively, the masses of $\mathrm{O}, \mathrm{C}$, and $\mathrm{X}$.

With $k_{1}=10, k_{2}=4.5, k_{3}=1.0, m_{1}=16, m_{2}=12$ and $m_{x}$ and $\theta$ chosen as indicated, Eq. (3) yields the maximum or carbonyl frequencies shown in Table I. The choice $k_{1}=10$ has been made to bring the result into the region of observed carbonyl frequencies for reasonable values of $\theta$. The chosen $k_{2}$ is an acceptable representation of a normal $\mathrm{C}-\mathrm{C}$ bond and the result is insensitive to the choice of $k_{3}$. The square root of the highest $\lambda$ consistent with Eq. (3) is multiplied by 1303 to give the frequency in wave numbers. Each column of Table I shows, for a chosen XCX angle (20), the frequency for $m_{x}$ equal to unity, 12,96 , and $\infty$.

The column for $120^{\circ}$, which should be most nearly representative of ordinary molecules, checks the results of Burkard in showing a relatively small frequency change for $m_{x}$ larger than 12 . The frequency is somewhat sensitive to the XCX angle, but this does not have a serious adverse effect because in most cases of interest the angle is known or can be estimated with fair accuracy. Smaller angles produce higher frequencies and an increased sensitivity to the mass of $\mathrm{X}$.

The maximum mass effect occurs in the linear model with zero XCX angle, but the effects at all angles are similar in form, and merely increase somewhat in magnitude as the angle decreases. For further consideration 
TABLE II. Frequencies of the linear model $M_{2} \mathrm{C}_{2} \mathrm{CO}$ as a function of the mass $M$.

\begin{tabular}{lcccc}
\hline \hline$M$ & 1 & 12 & 96 & $\infty$ \\
$\nu$ & 1879 & 1907 & 1903 & 1903 \\
\hline
\end{tabular}

it can safely be assumed that the effect of extending the model to $(M \mathrm{C})_{2} \mathrm{CO}$, when $m_{x}=12$, will also vary with the XCX angle in a similar manner and will reach its maximum at the zero angle. This assumption will be supported by numerical examples at a later point.

With the aid of this reasonable assumption, a limit can be obtained in a relatively simple manner for the effect of introducing detail into the $\mathrm{X}$ groups of the original model. The linear $\mathrm{C}_{2} \mathrm{CO}$ model (Table I, first column, second row, frequency 1888) is extended to a four particle model $M_{2} \mathrm{C}_{2} \mathrm{CO}$ in which a mass $2 M$ is attached to the doubled carbon mass by a doubled carbon-carbon bond of force constant 9. Table II shows the frequencies for four values of $M$. The equations used for this frequency calculation are omitted because their general utility is not evident. The first listed frequency is the second root of the cubic secular equation, while each of the other three is the highest root. Consequently the inference should not be made that the "carbonyl" frequency passes through a maximum in the range of $M$ between the first two tabulated values. Each root, the first and the second, decreases smoothly with increasing mass over the entire range. In the first interval $(1<M<12)$ the concept of a "carbonyl" frequency merely loses useful significance, since over a part of the range neither of the two upper roots of the secular equation can be justifiably identified with the carbonyl group.

Less sensitivity of the pertinent frequency to the mass $M$ is observed for this model, $M_{2} \mathrm{C}_{2} \mathrm{CO}$, than for the linear model $\mathrm{X}_{2} \mathrm{CO}$. The maximum root, however, gives frequencies which are 15 or more units higher than that of the linear $\mathrm{C}_{2} \mathrm{CO}$ (1888). An indication that the tabulated case represents the maximum modification of $\mathrm{C}_{2} \mathrm{CO}$ pertinent to the present problem can be obtained by examining two further cases. In the first, the mass $M_{2}$ is halved and attached as two opposed masses $M$ by bonds at right angles to the $\mathrm{C}_{2} \mathrm{CO}$ line. Only bending forces of the two $M \mathrm{C}$ bonds, for which the force constant is set at $10^{5}$ dynes $/ \mathrm{cm}$, are involved in the pertinent parallel vibration. The frequency for all values of $M$ from 1.0 to infinity lies between 1891 and 1893 wave numbers.

The second case is chosen to show that the effect of extending $\mathrm{C}_{2} \mathrm{CO}$ to $(M \mathrm{C})_{2} \mathrm{CO}$ decreases as the $\mathrm{CCC}$ angle increases. It is sufficient to calculate the frequency only for infinite $M$, which simplifies the fifth degree secular equation which would give the parallel frequencies with finite $M$ values. From this the behavior with smaller $M$ can be inferred accurately enough by analogy to the linear and T-shaped models just con- sidered. When infinite masses are attached to the two carbons of $\mathrm{C}_{2} \mathrm{CO}$, with the $\mathrm{CCC}$ angle at 90 degrees, by bonds parallel to the $\mathrm{CO}$ bond with the $M \mathrm{C}$ stretching force constant at 4.5 and the constant for relative lateral displacement of $\mathrm{C}$ and $M$ set at 1.0, the calculated carbonyl frequency is 1769 . If the infinite masses are attached by like bonds perpendicular to the $\mathrm{CO}$ bond, the frequency is 1766 . These can be compared with 1761 from Table I for the 90 degree $\mathrm{C}_{2} \mathrm{CO}$ model.

The effects would be slightly larger for smaller finite masses, but would tend to decrease if the $\mathrm{CCC}$ angle were increased toward $120^{\circ}$. The result supports the assumption that it is valid to draw qualitative conclusions from calculations based upon the models obtained by extending the linear $\mathrm{C}_{2} \mathrm{CO}$ system.

It has been shown by numerical examples that the maximum effect of extending the $\mathrm{C}_{2} \mathrm{CO}$ model by symmetrical coplanar attachment of $M$ to form $M_{2} \mathrm{C}_{2} \mathrm{CO}$ occurs for linear extension of the linear $\mathrm{C}_{2} \mathrm{CO}$, and is near 20 wave numbers. For CCC angles from 90 to 120 degrees the maximum effect of two similar coplanar extensions, obtained in part by inference, is of the order of 10 wave numbers. The effect of the substitution decreases as the angle between the $M \mathrm{C}$ and $\mathrm{CO}$ bonds increases and has become nearly negligible when these bonds are perpendicular.

The remaining unanswered questions are concerned with the effects of noncoplanar extension, and of simultaneous attachment of three masses to each outer carbon atom of the $\mathrm{C}_{2} \mathrm{CO}$ model. It is reasonable, and, if an answer is to be obtained in a practical manner, necessary, to assume that the effect of noncoplanar extension is of the same order as the coplanar effects. When one of three masses attached to each of the carbons is located for the maximum effect, the angles will be such that the influence of the other two is much smaller.

Consequently, even if the effects of adding three individual masses to each carbon are additive, the total influence should be less than twice that of single extension. These considerations lead to the conclusion that for fixed force constants and $\mathrm{CCC}$ angle the carbonyl frequency of a symmetrical ketone $\left(R_{3} \mathrm{C}\right)_{2} \mathrm{CO}$ should not vary more than about 25 wave numbers with changes in the mass and character of the $R$ groups. Since, by calculation and reasonable inference, the frequency of any symmetrical ketone lies within 15 wave numbers of the frequency calculated for $\mathrm{C}_{2} \mathrm{CO}$ when the $\mathrm{CCC}$ angles are the same and lie within reasonable limits, it follows that substitution of the $\mathrm{C}_{2} \mathrm{CO}$ model for the correct more detailed structure will give a correspondingly accurate evaluation of the force constant from the measured frequency.

When, for CCC angles from 90 to 120 degrees, the carbonyl force constants required to produce frequencies from 1600 to $1800 \mathrm{~cm}^{-1}$ by Eq. (3) are determined, with $k_{2}, k_{3}$ and the masses held constant, it is found 
that at constant angle the force constant is a nearly linear function of the frequency, and, at constant $k$ the relation between angle and frequency is also nearly linear. The relations are expressed by Eq. (4) which is given in two equivalent forms.

$$
\begin{gathered}
\nu\left(\mathrm{cm}^{-1}\right)=1278+68 k-2.2 \varphi \\
k=0.01471 \nu+0.03235 \varphi-18.794 .
\end{gathered}
$$

In Eq. (4) $k$ is the carbonyl force constant in practical units (actual $k \times 10^{-5}$ ) and $\varphi$ is the CCC angle in degrees. For angles from 90 to 120 degrees, with the appropriate force constants, frequencies from 1600 to $1800 \mathrm{~cm}^{-1}$ obtained with this equation are within 2 wave numbers of the values given by Eq. (3).

Force constants corresponding to the chosen range of frequencies vary from 7.7 to 11.6 practical units. It is probable, however, that the assumption of a constant normal carbon-carbon force constant limits the applicability of Eq. (4) to a relatively narrow range. This assumption can be expected to be accurate only for unconjugated ketones with "normal" CCC angles, since it is obvious that conjugation will change the nature of the carbon-carbon bond, and it seems reasonable to suppose that any considerable change of the angle will result in changes in the strength of both the carbonyl and the carbon-carbon bonds. Observed frequency differences for otherwise equivalent unconjugated ketones with appreciably different $\mathrm{CCC}$ angles will probably not be accurately calculated by Eq. (4) because the force constants may change at the same time.

The frequency range (1705-1720) corresponds to a range of 0.22 in the carbonyl force constant if the angle is assumed to be constant and the CC force constant is fixed at 4.5 practical units. Variation of the CCC angle from 109 to 120 degrees would increase the calculated force constant by 0.34 unit. Failure of the assumption of constant $\mathrm{CC}$ bond strength would introduce a relatively small additional uncertainty. It can be concluded that the stretching force constant of the carbonyl bond in unconjugated symmetrical ketones is approximately constant in the range $10.2 \pm 0.3$ practical units. Since the observed frequency range applies also to unsymmetrical ketones, and the factors which determine the force constant should not be sensitive to symmetry, this value should also be characteristic of unsymmetrical ketones.

The lower frequency (1686) observed by Stiles for $t$. butyl ketone is probably due primarily to a steric increase of the CCC angle, which may or may not be accompanied by a decrease in the force constant. With a fixed nearly normal CC bond strength, this frequency implies an increase of $10-17$ degrees in the bond angle at constant carbonyl force constant, a decrease of $0.32-0.55$ practical units in the force constant at constant angle, or some combination of the two effects.

Before it can be concluded that the force constant of a normal carbonyl bond in the absence of resonance effects is $10.2 \pm 0.3$, the possible effect of hyperconjugation should be considered. In this connection, it is of interest that $t$. butyl ketone, which should be unaffected by hyperconjugation, may have a lower force constant. Otherwise, the near constancy of the observed frequencies for unconjugated ketones with varying degrees of susceptibility to hyperconjugation suggests that the effect is negligible. An appreciable effect on the carbonyl bond, however, could occur if it were compensated, in determining the frequency, by an opposite effect on the CC bonds.

Equation (4) is evidently not applicable to aldehydes, although it is interesting that their frequencies fall in the same general range as the unconjugated ketones. It is of particular interest, however, that force constants calculated for the carbonyl bond in formaldehyde are from 20 to $50 \%$ higher, with a variety of potential functions, than the value derived here for the unconjugated ketones. The formaldehyde frequency, 1745, appears to be reasonably in line with the ketone frequencies, but, since the phase relations of the particle displacements are different from those in the ketone model, direct comparison of the frequencies is less significant than comparison of the force constants.

It seems evident, from the occurrence of carbonyl frequencies as low as 1600 in molecules with conjugation or resonance, that the carbonyl force constant can be considerably less than the unconjugated ketone value. There is, however, no clear way at present of determining how much less, since the problem is complicated by variable unknown force constants for the $\mathrm{CC}$ bonds of the model $\mathrm{C}_{2} \mathrm{CO}$.

In conclusion, there appears to be a fairly constant carbonyl force constant for unconjugated ketones at about $10.2 \pm 0.3\left(\times 10^{5}\right)$ dynes $/ \mathrm{cm}$. In formaldehyde and possibly also other aldehydes, the value is higher, while in conjugated ketones, lower values will appear. It is interesting that here, as well as with carboncarbon bonds, the most representative double bond force constant is near twice the value for the single bond. 